\title{
ANÁLISIS DE LAS GUÍAS DOCENTES DE LAS ASIGNATURAS DE CARTOGRAFÍA EN LOS ACTUALES GRADOS DE GEOGRAFÍA EN ESPAÑA
}

Juan F. Martínez Murilloํㄹ José Jesús Delgado Peña ${ }^{2}$

Recibido: 17/05/2019

Aceptado: 08/09/2019

\section{RESUMEN:}

Este trabajo realiza una descripción de la enseñanza de la Cartografía en los actuales estudios universitarios de grado relacionados con la Geografía. Se ha generado una base de datos que incluye 26 grados en total, en los que al menos se imparte una asignatura de Cartografía. El resultado del análisis arroja una situación algo dispar entre grados en Geografía, especialmente, y la necesidad de incrementar los estudios en Cartografía y las nuevas tecnologías para hacer frente a las competencias cartográficas que debe adquirir el futuro investigador, docente o profesional geógrafo/a.

\section{Palabras clave:}

Cartografía, Grado en Geografía, Enseñanza, Guía Docente.

\section{Abstract:}

This study deals with the teaching of Cartography in the current degrees related to Geography in the high education in Spain. A26-degrees database was compiled considering those where there was one subject in Cartography at least. The results show a very dissimilar condition, mainly, among the degree in Geography, as well as the necessity in increasing

\footnotetext{
1 Departamento de Geografía, Universidad de Málaga.jfmmurillo@uma.es

2 Departamento de Geografía, Universidad de Málaga.jdelgado@uma.es
} 
the presence of Cartography subjects to face the new and challenging competencies to be achieve by the future researchers, teachers, and professionals in Geography.

\section{KEYWORDS:}

Cartography, degree in geography, teaching, teaching guide.

\section{RESUMÉ:}

Ce travail décrit l'enseignement de la cartographie dans les programmes d'études universitaires en rapport avec la géographie. Une base de données contenant 26 degrés au total a été générée, dans laquelle au moins un sujet de cartographie est enseigné. Le résultat de l'analyse montre une situation quelque peu différente entre les diplômes de géographie, en particulier, et la nécessité d'accroître les études en cartographie et les nouvelles technologies pour faire face aux compétences cartographiques que le futur chercheur, enseignant ou géographe professionnel devrait acquérir.

\section{MoTS-CLÉ:}

Cartographie, diplôme de géographie, enseignement, guide de l'enseignment

\section{INTRODUCCIÓN}

Desde la década de los años 90 del pasado siglo, la enseñanza de la Geografía en el ámbito universitario se ha visto enriquecida por aportaciones didácticas y metodológicas, adoptando nuevas estrategias de enseñanza entre sus docentes. Ello es una respuesta al cambio tan rápido experimentado hacia la sociedad del conocimiento, lo cual afecta al modo en el que sus estudiantes aprehenden el espacio geográfico. Si a las tecnologías de la información y la comunicación (TIC), que ya desde finales de los ochenta se venían utilizando en ámbitos no universitarios (de Lázaro y Torres, 2012), se suman las Tecnologías de la de la Información Geográfica (TIG; a saber: sistemas de información geográfica, teledetección, cartografía digital, GPS, y la orto-fotogrametría), no cabe duda de que los y las estudiantes deben ser capaces de adquirir una serie de competencias en estas herramientas, básicas para la Cartografía actual, para poder responder a las demandas del mercado laboral y al mismo tiempo generar nuevas líneas de trabajo, con un espíritu crítico y constructivo.

El conocimiento detallado del territorio es una demanda clara de la sociedad dados los problemas ambientales y humanos a los que se enfrenta, en el contexto de Cambio Global actual. Según Luque Revuelto (2011, p. 184), en el tratamiento de los problemas que afectan al planeta, particularmente en los medioambientales, se puede apreciar una cierta unidad o consenso que consiste en aceptar que la forma más precisa y económica de estudiar, analizar o cuantificar estos problemas es desde el espacio. De esto se deriva la importancia de 
trabajar en el aula con todos aquellos recursos que permitan acercarnos al conocimiento de los hechos geográficos desde un perspectiva global como son las imágenes de los satélites, las técnicas de fotointerpretación o, en el caso que nos interesa, la cartografía digital.

El mapa, como documento cartográfico, continúa siendo una herramienta básica para el estudio, análisis y diagnóstico de esos problemas ambientales y sociales, así como para proponer soluciones o modos sostenibles de gestión territorial. Por ello, en los estudios de Geografía, desde el mapa y con el mapa se debería construir una mejor clase teórica; del mismo modo que ésta parecerá incompleta sin la expresión cartográfica. Los mapas se hacen necesarios en la clase de geografía, tanto como necesaria es la contribución de los geógrafos a su elaboración (Sancho Comins, 1996, p. 15-16). Tomando de referencia a este autor, el mapa encierra la complejidad del proceso dc ejecución y pretende hacer de puente entre la realidad, rica e insondable, y el usuario. Ambos aspectos convierten el mapa en un elemento de gran valor que precisa de cuidados extremos en su elaboración y utilización, y se vuelve enormemente eficaz cuando se cumplen en él una serie de cualidades. A nivel educativo no podemos darnos por satisfechos en cuanto al papel que está desempeñando, pues el aprendizaje de su elaboración por parte del o la estudiante del grado de Geografía (en cualquiera de sus denominaciones) requiere de una mayor profundización en el conocimiento de las destrezas y actitudes más allá del del uso habitual de los mapas en la clase.

Según el Libro Blanco de Geografía (ANECA, 2005, p. 128), el 17\% de los geógrafos profesionales asociaban su labor a la actividad de la Cartografía, desde el complejo proceso de producción de bases cartográficas de carácter digital de detalle (topografía, catastral, etc.) hasta la representación y visualización de datos estadísticos (con una fuerte componente de semiología gráfica), pasando por la cartografía temática especializada (de usos del suelo, geológica, hidrográfica, etc.). Entre las múltiples tareas que desarrollan los geógrafos en la producción cartográfica se encuentran: el diseño de documentos cartográficos; la planificación de vuelos para la obtención de fotografía aérea; la recopilación de datos de campo; la gestión de cartografías básicas, incluida la realización, revisión y actualización; la fotointerpretación; el control del proceso de edición; o la aplicación de técnicas de semiología gráfica para la visualización de datos, entre otras. De Lázaro y Torres (2012) indica en este sentido la importancia de la formación en TIG para los egresados de los títulos universitarios en Geografía, en combinación con el aprendizaje adecuado de las TIC. La adquisición de competencias espaciales junto a las digitales es una cuestión de relevancia para el aprendizaje del alumnado de geografía (Gómez Ruiz y de Lázaro y Torres, 2012), luego las asignaturas de cartografía deben promover claramente esta cuestión.

Ante esto cabe preguntarse: ¿reciben los alumnos y las alumnas de los diferentes grados en Geografía, de las universidades del Estado español, la preparación adecuada 
para enfrentarse a las tareas mencionadas?; ¿reúnen las actuales asignaturas relacionadas con la Cartografía de los actuales grados, los contenidos necesarios para que nuestro alumnado adquiera las competencias pertinentes para llevar a cabo con éxito tales tareas, especialmente teniendo en cuenta la imbricación definitiva que la Cartografía presenta hoy día con las Tecnologías de la Información Geográfica (TIG), dado que los documentos cartográficos se producen hoy día gracias a ellas? Por todo esto, el objetivo de este trabajo es realizar una descripción del estado de la cuestión en cuanto a la enseñanza de la Cartografía en los actuales grados en Geografía, así como en otros grados en los que se imparten materias geográficas y cartográficas, ofreciendo una información descriptiva de las guías docentes de las diferentes asignaturas implicadas en su enseñanza directa.

\section{METODOLOGÍA}

Para alcanzar los objetivos fijados en este trabajo, se acudió a consultar dos fuentes de información principales:

-Ministerio de Ciencia, Innovación y Universidades. En este organismo público se recabó información de todos los grados universitarios relacionados con la enseñanza de la geografía, por medio de su aplicación “QEDU” (Qué Estudiar y Dónde en la Universidad). A través de esta aplicación, se accedió a la información relativa de cuáles universidades ofertaban estudios superiores de Geografía que fueron las siguientes: Grado en Geografía; Grado en Geografía y Ordenación del Territorio; Grado en Geografía y Gestión del Territorio; Grado en Geografía, Ordenación del Territorio y Gestión del Medio Ambiente; Grado en Geografía y Medio Ambiente; y Grado en Geografía e Historia.

-Las webs oficiales de las universidades en donde se imparten los grados mencionados anteriormente, en las cuales se han podido recopilar los planes de estudio de dichos grados, así como las guías docentes de las asignaturas referidas a la enseñanza de la Cartografía. Las guías docentes consultadas corresponden a las disponibles de modo online, en cada una de las universidades, para el curso académico 2018-19.

A partir de ambas fuentes de información, se confeccionó una base de datos que recogía información acerca de: asignaturas relacionadas con la Cartografía, carácter de la asignatura, curso de impartición, créditos ECTS, y duración. Igualmente, mediante la consulta de las diferentes guías docentes, se obtuvo información acerca de los contenidos, las metodologías docentes de enseñanza y los sistemas de evaluación. Toda esta información ha sido utilizada para tratar de caracterizar las asignaturas seleccionadas a fin de poder realizar una valoración aproximada de la situación de la enseñanza de la Cartografía en los grados de geografía. La base de datos confeccionada se organiza en base a las universidades donde se imparten los diferentes grados de Geografía o algún grado conjunto de Geografía y otra disciplina (Historia, por ejemplo), en los que se 
incluyen alguna asignatura de Cartografía, describiendo a continuación las diferentes variables tomadas. Esta base de datos se elaboró en una hoja de cálculo del programa de Microsoft Office Excel 2016.

\section{LA CARTOGRAFÍA EN LOS GRADOS DE GEOGRAFÍA}

La Tabla $n^{\circ} 1$ recoge la información relativa a los grados universitarios de estudios de Geografía, en sus diferentes modalidades, impartidos en universidades españolas, en las que se constata al menos una asignatura de Cartografía; fueron excluidos aquellos grados en los que no se impartía ninguna asignatura relacionada con esta materia. En su gran mayoría, los grados que incluyen estas asignaturas son grados de enseñanza universitaria de Geografía: Grado en Geografía; Grado en Geografía y Ordenación del Territorio; Geografía y Gestión del Territorio; Grado en Geografía, Ordenación del Territorio y Gestión del Medio Ambiente; y Grado en Geografía y Gestión del Medio Ambiente. No obstante, de los 26 grados universitarios seleccionados finalmente, tres de ellos corresponden a grados en Geografía e Historia, en los que al menos se imparte una asignatura de Cartografía.

En total, se han contabilizado 37 asignaturas de Cartografía impartidas entre todos los grados. La variabilidad en cuanto al número de asignaturas de Cartografía impartidas por grado es alta: el 56,0\% de los grados incluye sólo una asignatura específica de Cartografía; el 32,0\% dos asignaturas; y solo el 4,0\% (1 grado) cuatro asignaturas. El carácter de las asignaturas corresponde a "Formación básica" (33,3\%), "Obligatoria" $(47,2 \%)$, "Troncal” (5,6\%) y "Optativa" $(13,9 \%)$. El número de créditos ECTS que corresponden a cada asignatura es en la mayoría de los casos de 6; tan solo hay una asignatura con 12 créditos ECTS de las 37 contabilizadas.

El curso en el que se imparte mayoritariamente estas asignaturas relacionadas con la enseñanza de la Cartografía es el primero, con una frecuencia relativa del 64,9\%, seguido de cuarto $(16,2 \%)$, tercero $(10,8 \%)$ y segundo $(8,1 \%)$. Las asignaturas impartidas en primer curso y segundo tienen un carácter general de formación básica u obligatoria, mientras que las de tercero y cuarto, optativas. Al igual que con los créditos ECTS, la impartición de las asignaturas es casi exclusivamente con un carácter temporal semestral; tan solo una de ellas, la referida con 12 ECTS, tiene carácter anual.

\begin{tabular}{|l|l|l|l|l|l|l|}
\hline Universidad & Grado & Asignaturas & Carácter & Curso & ECTS & Duración \\
\hline $\begin{array}{l}\text { Universitat } \\
\text { d'Alacant }\end{array}$ & Grado en Geografía & $\begin{array}{l}\text { Introducción a la } \\
\text { Cartografía y Formas } \\
\text { de Representación }\end{array}$ & Obligatoria & $1^{\circ}$ & 6 & Semestral \\
\hline $\begin{array}{l}\text { Universitat } \\
\text { Autónoma de } \\
\text { Barcelona }\end{array}$ & $\begin{array}{l}\text { Grado en Geografía } \\
\text { y Ordenación del } \\
\text { Territorio }\end{array}$ & Cartografía & $\begin{array}{l}\text { Formación } \\
\text { básica }\end{array}$ & $1^{\circ}$ & 6 & Semestral \\
\hline
\end{tabular}




\begin{tabular}{|c|c|c|c|c|c|c|}
\hline $\begin{array}{l}\text { Universidad } \\
\text { Autónoma de } \\
\text { Madrid }\end{array}$ & $\begin{array}{l}\text { Grado en Geografía } \\
\text { y Ordenación del } \\
\text { Territorio }\end{array}$ & $\begin{array}{l}\text { Introducción a la } \\
\text { Cartografía }\end{array}$ & $\begin{array}{l}\text { Formación } \\
\text { básica }\end{array}$ & $1^{\mathrm{o}}$ & 6 & Semestral \\
\hline \multirow[t]{2}{*}{$\begin{array}{l}\text { Universitat de } \\
\text { Barcelona }\end{array}$} & \multirow[t]{2}{*}{ Grado en Geografía } & Cartografía General & $\begin{array}{l}\text { Formación } \\
\text { básica }\end{array}$ & $1^{\circ}$ & 6 & Semestral \\
\hline & & Cartografía Temática & $\begin{array}{l}\text { Formación } \\
\text { básica }\end{array}$ & $2^{\circ}$ & 6 & Semestral \\
\hline \multirow[t]{2}{*}{$\begin{array}{l}\text { Universidad } \\
\text { de Cantabria }\end{array}$} & \multirow[t]{2}{*}{$\begin{array}{l}\text { Grado en Geografía } \\
\text { y Ordenación del } \\
\text { Territorio }\end{array}$} & $\begin{array}{l}\text { Geografía y } \\
\text { Tecnologías de la } \\
\text { Información y de la } \\
\text { Comunicación }\end{array}$ & $\begin{array}{l}\text { Formación } \\
\text { básica }\end{array}$ & $1^{\circ}$ & 6 & Semestral \\
\hline & & $\begin{array}{l}\text { Cartografía y } \\
\text { Técnicas de Análisis } \\
\text { Geográfico }\end{array}$ & $\begin{array}{l}\text { Formación } \\
\text { básica }\end{array}$ & $1^{\circ}$ & 6 & Semestral \\
\hline $\begin{array}{l}\text { Universidad } \\
\text { de Castilla-La } \\
\text { Mancha }\end{array}$ & $\begin{array}{l}\text { Grado en Geografía } \\
\text { y Ordenación del } \\
\text { Territorio }\end{array}$ & Cartografía* & Obligatoria & $1^{\circ}$ & 6 & Semestral \\
\hline \multirow[t]{2}{*}{$\begin{array}{l}\text { Universidad } \\
\text { Complutense } \\
\text { de Madrid }\end{array}$} & \multirow[t]{2}{*}{$\begin{array}{l}\text { Grado en Geografía } \\
\text { y Ordenación del } \\
\text { Territorio }\end{array}$} & $\begin{array}{l}\text { Cartografía y } \\
\text { Técnicas de } \\
\text { Representación I }\end{array}$ & Obligatoria & $1^{\mathrm{o}}$ & 6 & Semestral \\
\hline & & $\begin{array}{l}\text { Cartografía y } \\
\text { Técnicas de } \\
\text { Representación II }\end{array}$ & Optativa & $3^{\circ}$ y $4^{\circ}$ & 6 & Semestral \\
\hline $\begin{array}{l}\text { Universidad } \\
\text { de } \\
\text { Extremadura }\end{array}$ & $\begin{array}{l}\text { Grado en Geografía } \\
\text { y Ordenación del } \\
\text { Territorio }\end{array}$ & $\begin{array}{l}\text { Cartografía y } \\
\text { Fotointerpretación }\end{array}$ & Obligatoria & $1^{\mathrm{o}}$ & 6 & Semestral \\
\hline $\begin{array}{l}\text { Universitat de } \\
\text { Girona }\end{array}$ & $\begin{array}{l}\text { Grado en Geografía, } \\
\text { Ordenación del } \\
\text { Territorio y Gestión } \\
\text { del Medio Ambiente }\end{array}$ & $\begin{array}{l}\text { Anàlisi i tractament } \\
\text { de la informació } \\
\text { geogràfica }\end{array}$ & Obligatoria & $1^{\mathrm{o}}$ & 12 & Anual \\
\hline \multirow[t]{2}{*}{$\begin{array}{l}\text { Universidad } \\
\text { de Granada }\end{array}$} & \multirow{2}{*}{$\begin{array}{l}\text { Grado en Geografía } \\
\text { y Gestión del } \\
\text { Territorio }\end{array}$} & \begin{tabular}{|l} 
Principios de \\
Cartografía
\end{tabular} & Obligatoria & $1^{\circ}$ & 6 & Semestral \\
\hline & & $\begin{array}{l}\text { Cartografía Digital y } \\
\text { Temática }\end{array}$ & Obligatoria & $3^{\circ}$ & 6 & Semestral \\
\hline \multirow{2}{*}{$\begin{array}{l}\text { Universitat } \\
\text { de les Illes } \\
\text { Balears }\end{array}$} & \multirow[t]{2}{*}{ Grado en Geografía } & Cartografía & $\begin{array}{l}\text { Formación } \\
\text { básica }\end{array}$ & $1^{\mathrm{o}}$ & 6 & Semestral \\
\hline & & $\begin{array}{l}\text { Tècniques de } \\
\text { Disseny Cartogràfic } \\
\text { i Cartografia } \\
\text { Automàtica }\end{array}$ & $\begin{array}{l}\text { Formación } \\
\text { básica }\end{array}$ & $1^{\circ}$ & 6 & Semestral \\
\hline $\begin{array}{l}\text { Universidad } \\
\text { de La Laguna }\end{array}$ & $\begin{array}{l}\text { Grado en Geografía } \\
\text { y Ordenación del } \\
\text { Territorio } \\
\end{array}$ & $\begin{array}{l}\text { Expresión Gráfica y } \\
\text { Cartográfica }\end{array}$ & $\begin{array}{l}\text { Formación } \\
\text { básica }\end{array}$ & $1^{\mathrm{o}}$ & 6 & Semestral \\
\hline $\begin{array}{l}\text { Universidad } \\
\text { de La Rioja }\end{array}$ & $\begin{array}{l}\text { Grado en Geografía } \\
\text { e Historia }\end{array}$ & $\begin{array}{l}\text { Cartografía y } \\
\text { Representación } \\
\text { Gráfica } \\
\end{array}$ & Obligatoria & $4^{\circ}$ & 6 & Semestral \\
\hline
\end{tabular}


Análisis de las guías docentes de las asignaturas de Cartografía en los actuales Grados...

\begin{tabular}{|c|c|c|c|c|c|c|}
\hline $\begin{array}{l}\text { Universidad } \\
\text { de Las Palmas } \\
\text { de Gran } \\
\text { Canarias }\end{array}$ & $\begin{array}{l}\text { Grado en Geografía } \\
\text { y Ordenación del } \\
\text { Territorio }\end{array}$ & $\begin{array}{l}\text { Cartografía básica en } \\
\text { Geografía }\end{array}$ & $\begin{array}{l}\text { Formación } \\
\text { básica }\end{array}$ & $1^{\circ}$ & 6 & Semestral \\
\hline \multirow[t]{2}{*}{$\begin{array}{l}\text { Universidad } \\
\text { de León }\end{array}$} & \multirow{2}{*}{\begin{tabular}{|l} 
Grado en Geografía \\
y Ordenación del \\
Territorio
\end{tabular}} & $\begin{array}{l}\text { Cartografía I: Lectura } \\
\text { Básica de Mapas }\end{array}$ & Obligatoria & $1^{\circ}$ & 6 & Semestral \\
\hline & & Cartografía II & Obligatoria & $2^{\circ}$ & 6 & Semestral \\
\hline \multirow[t]{2}{*}{$\begin{array}{l}\text { Universitat de } \\
\text { Lleida }\end{array}$} & \multirow[t]{2}{*}{ Grado en Geografía } & $\begin{array}{l}\text { Introducción a la } \\
\text { Cartografía }\end{array}$ & Troncal & $1^{\circ}$ & 6 & Semestral \\
\hline & & $\begin{array}{l}\text { Cartografía Temática } \\
\text { y SIG }\end{array}$ & Troncal & $1^{\circ}$ & 6 & Semestral \\
\hline \multirow[t]{2}{*}{$\begin{array}{l}\text { Universidad } \\
\text { de Málaga }\end{array}$} & \multirow{2}{*}{$\begin{array}{l}\text { Grado en Geografía } \\
\text { y Gestión del } \\
\text { Territorio }\end{array}$} & $\begin{array}{l}\text { Principios de } \\
\text { Cartografía } \\
\end{array}$ & $\begin{array}{l}\text { Formación } \\
\text { básica }\end{array}$ & $1^{\circ}$ & 6 & Semestral \\
\hline & & Cartografía Temática & Obligatoria & $3^{\circ}$ & 6 & Semestral \\
\hline \multirow{2}{*}{$\begin{array}{l}\text { Universidad } \\
\text { de Murcia }\end{array}$} & \multirow{2}{*}{$\begin{array}{l}\text { Grado en Geografía } \\
\text { y Ordenación del } \\
\text { Territorio }\end{array}$} & Cartografía I & Obligatoria & $1^{\circ}$ & 6 & Semestral \\
\hline & & Cartografía II & Obligatoria & $1^{\circ}$ & 6 & Semestral \\
\hline UNED & $\begin{array}{l}\text { Grado en Geografía } \\
\text { e Historia }\end{array}$ & Cartografía & Optativa & $5^{\circ}$ & 6 & Semestral \\
\hline \multirow[t]{4}{*}{$\begin{array}{l}\text { Universidad } \\
\text { de Oviedo }\end{array}$} & \multirow{4}{*}{$\begin{array}{l}\text { Grado en Geografía } \\
\text { y Ordenación del } \\
\text { Territorio }\end{array}$} & $\begin{array}{l}\text { Introducción a la } \\
\text { Cartografía }\end{array}$ & $\begin{array}{l}\text { Formación } \\
\text { básica }\end{array}$ & $1^{\circ}$ & 6 & Semestral \\
\hline & & Cartografía Temática & Obligatoria & 3 & 6 & Semestral \\
\hline & & $\begin{array}{l}\text { Cartografía del } \\
\text { Medio Urbano }\end{array}$ & Optativa & $4^{\circ}$ & 6 & Semestral \\
\hline & & \begin{tabular}{|l|} 
Cartografía del \\
Medio Natural \\
\end{tabular} & Optativa & $4^{\circ}$ & 6 & Semestral \\
\hline $\begin{array}{l}\text { Universidad } \\
\text { Pablo de } \\
\text { Olavide } \\
\end{array}$ & $\begin{array}{l}\text { Grado en Geografía } \\
\text { e Historia }\end{array}$ & Cartografía Histórica & Optativa & $4^{\circ}$ & 6 & Semestral \\
\hline $\begin{array}{l}\text { Universidad } \\
\text { del País Vasco }\end{array}$ & $\begin{array}{l}\text { Grado en Geografía } \\
\text { y Ordenación del } \\
\text { Territorio } \\
\end{array}$ & Cartografía Básica & Obligatoria & $1^{\circ}$ & 6 & Semestral \\
\hline $\begin{array}{l}\text { Universidad } \\
\text { de Salamanca }\end{array}$ & Grado en Geografía & Cartografía & Obligatoria & $1^{\circ}$ & 6 & Semestral \\
\hline \multirow[t]{2}{*}{$\begin{array}{l}\text { Universitat de } \\
\text { València }\end{array}$} & \multirow[t]{2}{*}{$\begin{array}{l}\text { Grado en Geografía } \\
\text { y Medio Ambiente }\end{array}$} & Cartografía I & $\begin{array}{l}\text { Formación } \\
\text { básica }\end{array}$ & $1^{\circ}$ & 6 & Semestral \\
\hline & & Cartografía II & Obligatoria & $2^{\circ}$ & 6 & Semestral \\
\hline $\begin{array}{l}\text { Universidad } \\
\text { de Vigo }\end{array}$ & $\begin{array}{l}\text { Grado en Geografía } \\
\text { e Historia }\end{array}$ & $\begin{array}{l}\text { Sistema de } \\
\text { Representación } \\
\text { Geográfica } \\
\end{array}$ & Obligatoria & $3^{\circ}$ & 6 & Semestral \\
\hline
\end{tabular}

*, impartida en el curso 2017-18.

TABLA $\mathrm{N}^{\mathrm{0}} 1$. Información sobre las universidades que ofertan grados en geografía, en sus diferentes modalidades, en los cuales se imparte alguna asignatura de Cartografía. Fuente: Ministerio de Educación y Formación Profesional y elaboración propia. 


\section{ANÁLISIS DE LAS GUÍAS DOCENTES}

A continuación, se realiza un análisis del contenido temático de las guías docentes de las asignaturas de cartografía seleccionadas. Este análisis se va a realizar atendiendo a, si en el grado en cuestión, se imparte solo una asignatura de cartografía o varias, de manera que puede diferenciarse asignaturas de corte básico o fundamental y de perfil más específico, es decir, de cartografía temática; no obstante, cabe señalar que, en aquellos grados en los que solo hay una asignatura de Cartografía, esta incluye no solo aspectos fundamentales, sino también específicos de cartografía temática.

\subsection{Los contenidos}

Las asignaturas de corte básico o fundamental aparecen en todos los grados seleccionados. Estas suelen tener por objetivo introducir al alumnado en el mundo de la cartografía, su importancia para la sociedad y en la profesión de geógrafa/o. A este objetivo genérico, se unen otros más específicos que se encuentran directamente relacionados con los contenidos temáticos de las asignaturas.

La cartografía básica o fundamental se estructura en unos contenidos o temas fundamentales que, en general, pueden agruparse en los siguientes:

1) Introducción a la Cartografía. Se trata de un tema básico, en el que se introduce al alumnado en la materia de cartografía dado que, generalmente, es la primera vez que se enfrentan a este tipo de conocimiento; de manera habitual, en educación secundaria, es una materia poco o nada tratada por el profesorado, aunque con excepciones. Este tema está presente en todas las guías docentes consultadas de un modo u otro, con el objetivo de acercar al alumnado a una materia que es fundamental en la formación y perfil profesional del geógrafo.

2) Evolución histórica de la Cartografía. A veces como tema independiente, a veces incluido en el tema anterior, este tema no siempre está presente en las guías docentes consultadas. Se trata de un tema cuyos objetivos están dirigidos a ofrecer al alumnado una visión histórica de la cartografía, generalmente, desde la Antigüedad, hasta nuestros días, poniendo énfasis en la importancia de la cartografía en la sociedad de cada momento.

3) Fundamentos de la Cartografía. Bajo esta denominación general, señalamos la presencia de una serie de temas básicos para el aprendizaje de la Cartografía que se incluyen en todas las guías de asignaturas consultadas. Se trata de temas que están dirigidos a que el alumnado aprenda las bases fundamentales en la elaboración e interpretación de Cartografía, especialmente, las relacionadas con: la forma de la Tierra; la problemática de pasar de una superficie curva a una plana; los sistemas de proyección y de coordenadas; la escala y la medición de distancias y superficies; tipología de documentos y fuentes 
Análisis de las guías docentes de las asignaturas de Cartografía en los actuales Grados...

cartográficas; características y elementos del documento cartográfico y su interpretación, finalidad y uso, entre otros aspectos.

4) Cartografía básica. En este aspecto, las asignaturas fundamentales de Cartografía hacen hincapié en aumentar las destrezas de manejo e interpretación de la información geográfica en mapas topográficos, siendo el más trabajado el Mapa Topográfico Nacional a escala 1:50.000. Básicamente, son tres los aspectos a los que se atiende: la comprensión del relieve por medio de las curvas de nivel, la interpretación del medio físico y del medio humano, así como de las interrelaciones existentes entre ambos.

5) Cartografía temática. En la mayoría de las guías docentes consultadas de asignaturas de Cartografía básica o fundamental, aparece un tema ligado a la Cartografía temática, independientemente de que haya o no otras asignaturas más específicas al respecto, en algún curso posterior. Se da especial énfasis a los tipos de mapas construidos con puntos, líneas y polígonos, así como a la representación de variables mediante coropletas, isolíneas, etc., e incluso gráficos.

6) Cartografía y TIG. En este caso, se trata de temas con contenidos relacionados con la evolución reciente de la cartografía, el paso del formato papel al digital, de los Sistemas de Información Geográfica (SIG) a los servidores Web, la Directiva europea INSPIRE, etc. En varias ocasiones, se hace una introducción a los SIG, especialmente, iniciando al alumnado en la cartografía derivada de la digitalización vectorial y en la cartografía numérica o en formato ráster. También, pueden consultarse guías que incluyen las tecnologías más recientes para la producción de cartografía, como el LTSLIDAR y la fotogrametría automatizada (SfM-MVS) terrestre y a partir de vehículos aéreos no tripulados.

7) Por último, en algunas de las guías docentes consultadas, aparecen temas más innovadores o que no aparecen en otras guías. En este aspecto, destacan aquellas que incluyen un desarrollo temático más amplio ligado a la generación y producción del documento cartográfico, a la semiología o, incluso, a las diferentes normativas que deben cumplir los mapas en materia territorial, urbanística y ambiental.

En buena parte de las asignaturas de Cartografía General, los contenidos siguen una secuenciación más o menos similar, comenzando por una introducción a la materia, seguida habitualmente por una descripción de su evolución histórica, para entrar a continuación en temas más específicos sobre los fundamentos técnico-matemáticos y geográficos de los documentos cartográficos y sus tipos. No sería desdeñable ampliar estos contenidos con las nuevas tendencias en Cartografía, como la denominada Cartografía Crítica, ajena a la realizada por organismos oficiales gubernamentales, que pone en cuestión la realidad geográfica que ofrecen estos a través de los documentos cartográficos que generan (Sánchez y Pérez, 2014) combinación efectiva de nuevas 
prácticas cartográficas y enjuiciamiento de las existentes (Crampton y Krygier, 2006, en Canosa Zamora y García Carballo, 2017).

También sería interesante el estudio de lo que se llama cartografía colaborativa, muy en boga con el desarrollo de la web 2.0, y y donde los usuarios pasan de ser consumidores a prosumidores. En este sentido, el papel activo que toma el ciudadano, o es estudiante, para el uso de la cartografía como instrumento de reinvindicación social, por ejemplo, puede conllevar actividades y uso de recursos más en línea con la sociedad actual. En este sentido, «la web 2.0 en sí comparte en su esencia los mismos principios de la cartografía participativa, puesto que ambas postulan la participación activa de los ciudadanos y el que puedan expresar y difundir sus propias ideas, intereses y demandas», contribuyendo de esta forma al empoderamiento ciudadano (Subires, 2012: 208). Esta potencialidad podría trasladarse al aula desde una perspectiva metodológica.

En algún caso de las asignaturas analizadas, no se sigue el perfil clásico de Cartografía fundamental y temática, sino que se plantea una asignatura que, por ejemplo, integra contenidos relativos a las TIG, la información geográfica en la web, las bases de datos, el análisis estadístico, entre otros. También, se observa que la asignatura incluye tan solo aspectos históricos de la Cartografía, al estar incluida en un grado de historia o de humanidades, así como la preeminencia de contenidos ligados a los SIG, sin una presencia explícita de contenidos puramente cartográficos, como los enunciados en puntos anteriores.

En algunos grados, se incluye al menos otra asignatura más relacionada con la Cartografía, en este caso, dirigida a ampliar conocimientos en Cartografía Temática, desarrollando los aspectos más aplicados de la misma. En general, estas asignaturas articulan sus contenidos alrededor de los siguientes aspectos:

-El lenguaje cartográfico, la semiología, las variables visuales, la rotulación, los criterios de representación, así como la técnica necesaria para confeccionar documentos cartográficos correctos y legibles.

-El conocimiento y generación de los tipos de cartografía temáticas, cualitativas y cuantitativas, así como la necesaria para el trabajo de campo, en entornos SIG.

-La elaboración de proyectos cartográficos, el manejo de infraestructuras de datos espaciales, la generación de cartografía en entornos virtuales y/o interactivos.

-Y, por último, temas más específicos de cartografía temática especializada y aplicada al ámbito de la Geografía Física, Geografía Humana, y la Planificación Territorial.

En general, a priori, se puede decir que el alumnado de los distintos grados en Geografía que se imparten en las universidades españolas, reciben una formación en Cartografía fundamental o básica que atiende los conocimientos teóricos, técnicos y prácticos mínimos que deben adquirir para poder desarrollar las competencias pertinentes al respecto: 
básicamente, ser capaces de adquirir información geográfica, transformarla y sintetizarla para poder representarla gráfica y cartográficamente atendiendo a unos estándares de calidad técnica y semiológica, consiguiendo unos documentos cartográficos correctos para su uso en la investigación, la docencia, y/o la planificación y gestión territorial.

Sin embargo, en las asignaturas consultadas, los contenidos ligados a aspectos más recientes y avanzados desde el punto de vista tecnológicos, relacionados con la Cartografía, no aparecen demasiado desarrollados, salvo alguna excepción. En este sentido, es escaso el posible conocimiento que adquiere el alumnado de Cartografía sobre las nuevas tecnologías, como, por ejemplo, aquellas ligadas al LIDAR, tanto desde estaciones totales como desde vehículos aéreos no tripulados, así como el aprendizaje de programación (dado que en la mayoría de los casos, los SIG ya están implementados como herramientas de aprendizaje en estas asignaturas). Y existen más avances tecnológicos que pueden tener cabida en mayor o. menor grado en la cartografía digital como pueden ser la realidad aumentada o los escenarios virtuales. Es decir, la formación cartográfica del alumnado de los grados en Geografía es de tal punto incompleta, considerando los constantes avances tecnológicos en la producción de documentos cartográficos. No obstante, no sería aconsejable incluir todos estos nuevos conocimientos y técnicas en las asignaturas de Cartografía fundamental o básicas, dado que se trata de la primera vez (o casi) en la que el alumno o la alumna se enfrenta a tal tipo de conocimiento. Sin embargo, sí sería óptimo y útil de cara a su futuro investigador y/o profesional la inclusión de nuevas asignaturas más específicas, dirigidas a dar un nivel de conocimiento y de competencia más conectado con esas nuevas tecnologías. Es posible que estas nuevas tecnologías sean objeto de un estudio más concreto en otro tipo de asignaturas, especialmente, aquellas ligadas a los SIG, pero deberían estar ligadas aún más a asignaturas específicamente cartográficas. Caso aparte son aquellos alumnos/as de otros grados, como Historia o Humanidades, en los que se incluye una asignatura de cartografía, en cuyo caso sí está más ligada a darles una visión global e histórica de esta disciplina.

\subsection{Metodologías docentes y sistemas de evaluación}

En cuanto a la metodología docente y las actividades llevadas a cabo, se puede apuntar lo siguiente en lo que concierne a las asignaturas de Cartografía básica, en general, troncales u obligatorias, de primer curso. La Figura. 1 recoge la frecuencia absoluta de las diferentes metodologías docentes recopiladas en las guías docentes. En casi todas ellas, esta metodología docente pone en marcha dos grandes grupos de actividades principales: uno, la explicación del contenido teórico y otro, el desarrollo del contenido práctico. El primero se desarrolla básicamente a partir de las denominadas clases magistrales o lecciones expositivas. El segundo grupo ofrece una mayor variedad de actividades 
formativas, tanto presenciales como no presenciales; destacan las siguientes: ejercicios prácticos en el aula y/o en el laboratorio o aula de informática, trabajo de campo, trabajo individual, asistencia y participación en seminarios, entre otros. La estrategia docente para el desarrollo de estas prácticas no siempre viene bien descrita, pero en general podría decirse que suelen ponerse en práctica, al modo clásico, mediante la elaboración de ejercicios y trabajos individuales. En algunos casos, sí se indica más explícitamente que el desarrollo de este contenido práctico se efectúa mediante la aplicación de otras técnicas como el estudio de casos. Cabe reseñar también que no en todas las asignaturas hay trabajo de campo; tan solo en 7 de 28 asignaturas aparece como actividad formativa.

\section{Cartográfica básica}

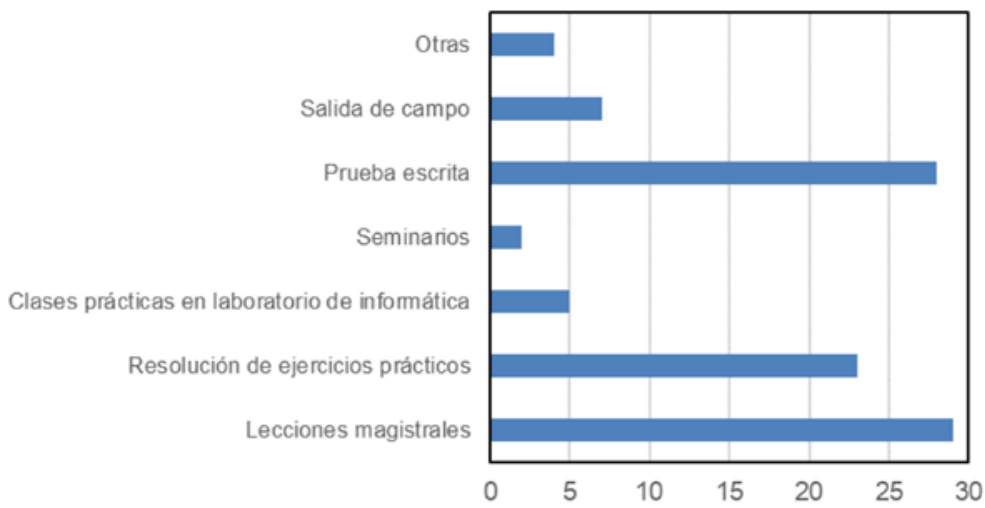

Cartografía Temática

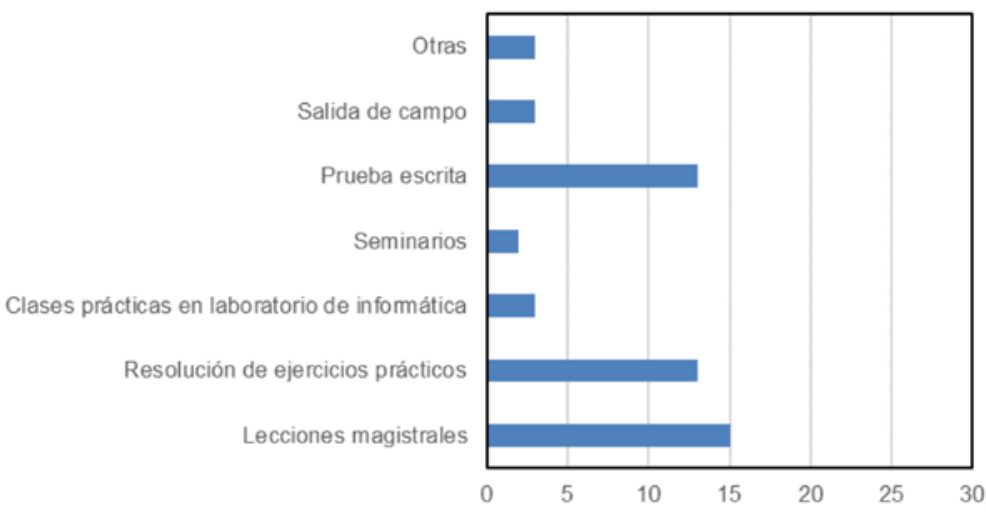

FiguRA. 1. Distribución absoluta de las metodologías docentes empleadas en la enseñanza de las asignaturas de Cartográfica básica y temática. 
Los sistemas de evaluación ofrecen también cierta homogeneidad a nivel general, siendo clara la utilización de los dos tipos de sistemas más comunes: la evaluación continua y la evaluación final mediante una prueba escrita. Sin embargo, en lo que sí se observa heterogeneidad es en el reparto de los porcentajes que suponen ambos sistemas en el $100 \%$ de la calificación final de la asignatura, en este caso, de cartografía básica (Figura. 2). El 34,5\% de las asignaturas otorgan más peso en la ponderación de la calificación final al examen o prueba escrita final, pero también pueden enumerarse con el mismo porcentaje aquellas en las que es la evaluación continua la que más influencia tiene. En el resto, con un 31,0\%, ambos sistemas de calificación aparecen con la misma ponderación del $50 \%$.

\section{Cartografía básica}

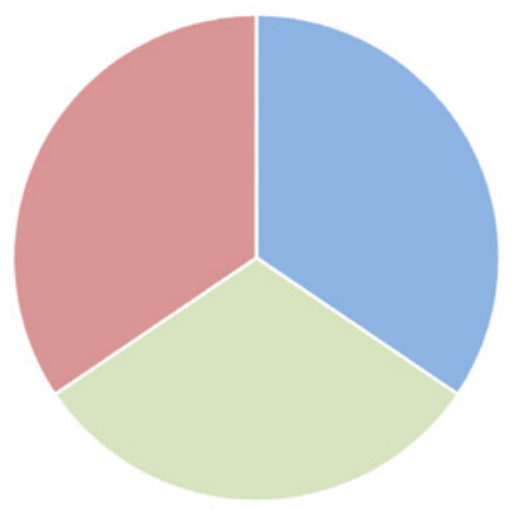

Cartografía temática

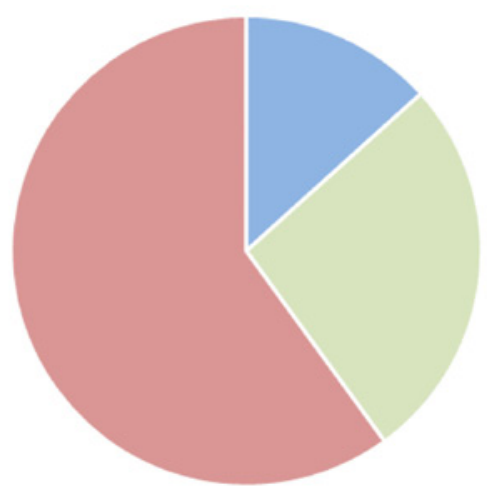

FIGURA. 2. Distribución relativa del paso otorgado a los sistemas de evaluación final y continua en las asignaturas de cartografía básica y temática.

También han sido analizadas las asignaturas de Cartografía que se imparten en cursos superiores a primero, en algunos casos obligatorias, en otros optativas, que tienen como objetivo dotar al alumno o alumna de conocimientos más específicos sobre Cartografía Temática y sus aplicaciones. Las metodologías docentes registradas en el análisis de las guías docentes no difieren demasiado de aquéllas de Cartografía básica: clases magistrales; clases prácticas con resolución de problemas, bien en el aula normal, bien en el aula o laboratorio de informática; participación en seminarios y salidas de campo; y realización de un trabajo, generalmente individual. En lo que sí se observa más diferencias es en el sistema de evaluación. El 64,3\% de las asignaturas consultadas otorga una mayor ponderación al sistema de evaluación continua, llegando en dos de ellas a suponer el $100 \%$ de la calificación final. A continuación, con un 
$28,6 \%$ están aquellas donde es igualitaria el peso dado a la evaluación de tipo continua y final. Por último, sólo en el 14,3\% de los casos, es la prueba final o examen la que aporta un mayor porcentaje a la calificación final de la asignatura.

\section{CONCLUSIONES}

Tras la consulta y análisis de 47 guías docentes relacionadas con la enseñanza de la Cartografía en los 26 grados seleccionados, específicos en Geografía, pero también en Geografía e Historia, y Humanidades, se establecen las siguientes conclusiones:

1) Al menos una asignatura de Cartografía básica se incluye en los grados en Geografía; no siempre en los de Geografía e Historia y en los de Humanidades. En general, son asignaturas cuatrimestrales, de primer curso, con 6 ECTS. En el caso de las asignaturas de Cartografía Temática, éstas no aparecen en todos los grados en Geografía, reduciéndose la formación cartográfica del alumnado de estos grados, algo que es fundamental en su posterior labor investigadora y profesional como geógrafos.

2) Los contenidos de las asignaturas fundamentales de Cartografía otorgan al alumnado una visión muy amplia del documento cartográfico, de su evolución histórica, sus bases científicas, artísticas y técnicas, la lectura e interpretación de los mapas topográficos, y de los principales mapas temáticos. En el caso de las asignaturas de Cartografía Temática, estas abundan en aspectos semiológicos y de representación cartográfica adecuada, y la selección del tipo de mapa en función de las variables analizadas, entre otros.

3) No existe una presencia uniforme y estructurada de la Cartografía en los estudios de Geografía en el ámbito universitario español, lo cual puede cercenar las competencias en esta materia que deba adquirir un futuro geógrafo o geógrafa, para convertirse en un profesional referente en esta disciplina, que es básica para la planificación territorial, ambiental y urbanística. Sería recomendable llevar a cabo una ampliación en asignaturas relacionadas con la Cartografía en los grados, especialmente, en los de Geografía, siendo bienvenida la inclusión de contenidos que den una base sólida de conocimientos en las nuevas tecnologías para la generación de productos cartográficos.

4) Se hace necesaria una reflexión en torno a la introducción de nuevas perspectivas dentro de la cartografía, como pueden ser la cartografía crítica o la cartografía colaborativa, tanto como instrumentos de mejora de la metodología y recursos docentes, como medios para aumentar la motivación del alumnado a la vez que se les ofrece una formación más relacionada con su día a día, una formación en definitiva más acorde con la sociedad actual en la que deben desarrollarse como profesionales y como ciudadanos.

5) También es importante incluir, especialmente en los cursos superiores, avances tecnológicos de la mano de las TIC que puedan mejorar su formación de cara a la futura 
vida profesional, como pueden ser, p. ejemplo, la tecnología LIDAR o la creación de escenarios virtuales.

6) Finalmente, desde el punto de vista metodológico y de los sistemas de evaluación, es recomendable aumentar la diversidad, fomentando las competencias básicas necesarias para el futuro desempeño profesional con actividades basadas en la realidad, de carácter eminentemente práctico, realizadas en equipo de manera colaborativa, y donde también se valoren los procesos y no exclusivamente los resultados.

\section{BIBLIOGRAFÍA}

Agencia Nacional de Evaluación de la Calidad y Acreditación, 2005. Libro Blanco del Títutlo de Grado en Geografía y Ordenación del Territorio. Madrid, ANECA.

Canosa Zamora, E., García Carballo, A., 2017. "Cartografías críticas de la ciudad". Treballs de la Societat Catalana de Geografia, 84, 145-160.

De Lázaro y Torres, M.L., 2012. "Las tecnologías de la información y la comunicación (TIC) en el proceso de enseñanza-aprendizaje de la geografía", en V. Gozálvez Pérez y J.A. Marco Molina (eds.), Geografía. Retos ambientales y territoriales. Actas del XXII Congreso de Geógrafos Españoles, Universidad de Alicante, 279-286.Gómez Ruiz, M.L., de Lázaro y Torres, M.L., González González, M.J., 2012. "Impulsar las competencias espaciales y digitales a través de un viaje virtual por Getafe", en Rafael de Miguel González, María Luisa de Lázaro y Torres, María Jesús Marrón Gaite (eds.), La educación geográfica digital, Universidad de Zaragoza, 471-484.

Luque Revuelto, R.M., 2011. "El uso de la cartografía y la imagen digital como recurso didáctico en la enseñanza secundaria. algunas precisiones en torno a Google Earth". Boletín de la Asociación de Geógrafos Españoles, 55, 183-210.

Sánchez, R., Pérez, A., 2014. "Mapeo 2.0. Ampliando los límites de la cartografía crítica". Ecología Política, 48, 24-27.

Sancho Comins, J.F., 1996. "El mapa como recurso didáctico en la enseñanza y aprendizaje de la geográfia". Didáctica de la Geografía, 1, 15-20.

Subires Mancera, María Purificación, 2012: “Cartografía participativa y web 2.0: Estudio de interrelaciones y análisis de experiencias”. Vivat Academia, 117, 201-216, 
